\title{
Development and Validation of Nomograms to Predict Overall Survival Outcomes in Serous Ovarian Cancer Patients with Satisfactory Cytoreductive Surgery and Chemotherapy
}

\author{
Yuan-Yuan Liu ${ }^{l, 2}$ \\ Ren-Feng Zhao ${ }^{2}$ \\ Chao Liu $^{2}$ \\ Jie Zhou ${ }^{2}$ \\ Liu Yang ${ }^{2}$ \\ Li Li (iD' \\ 'Department of Gynecologic Oncology, \\ Guangxi Medical University Cancer \\ Hospital, Key Laboratory of Early \\ Prevention and Treatment for Regional \\ High Frequency Tumor, Ministry of \\ Education, Nanning, Guangxi, People's \\ Republic of China; ${ }^{2}$ The People's Hospital \\ of Guangxi Zhuang Autonomous Region, \\ Nanning, Guangxi, People's Republic of \\ China
}

Correspondence: Yuan-Yuan Liu; Li Li Tel +86-I3877I 52206; +86-I3878II3406 Email 43322045@qq.com; yuanyuan_liu2021@I63.com

\begin{abstract}
Objective: Nomograms are statistics-based predictive tools that integrate predictive factors. Herein, a nomogram was developed and validated to predict the overall survival (OS) in serous ovarian cancer (SOC).

Methods: Primary SOC patients with satisfactory cytoreductive surgery, chemotherapy, and OS $\geq 1$ month were included in this study. A total of 6957 patients from the Surveillance, Epidemiology, and End Results (SEER) database comprised the training group and 1244 patients comprised the external validation group. The nomogram was structured on Cox models and evaluated in both the training and validation groups using consistency index, area under the receiver operating characteristics curve, calibration plots, and risk subgroup classification. Kaplan-Meier curves were plotted to compare the survival outcomes between subgroups. A decision-curve analysis was used to test the clinical value of the nomogram.

Results: Independent factors, including age, tumor grade, and Federation of Gynecology and Obstetrics (FIGO) stage, identified by multivariate analysis in the training cohort, were selected for the nomogram. The consistency indexes for OS were 0.689 in the training cohort and 0.639 in the validation cohort. The calibration curves showed good consistency between predicted and actual 3- and 5-year OS. Significant differences were observed in the survival curves of different risk subgroups. The decision-curve analysis indicated that our nomogram was superior to the American Joint Committee on Cancer (AJCC) staging system. Conclusion: A nomogram was constructed to predict the long-term OS in SOC and verified in Asians. The accurate predictions facilitated personalized treatments and follow-up strategies.
\end{abstract}

Keywords: nomograms, serous ovarian cancer, overall survival, SEER, external verification

\section{Introduction}

Epithelial ovarian cancer (EOC) is one of the most common malignant tumors of the female genital organs. As a subtype of EOC, serous ovarian cancer (SOC) presents a distinct biological profile from other histological types. ${ }^{1}$ In 2002 , Singer et al proposed a two-level classification system for SOC, wherein patients were divided into two subtypes: low-grade ovarian serous cancer (LGOS) and high-grade ovarian serous cancer (HGOS). ${ }^{1}$ In SOC, the binary theory of low- and high-grade disease is widely accepted because of the obvious differences in tumorigenesis, malignant biological behaviors, and molecular characteristics. ${ }^{2}$ 
HGOS is a type II and the most common type of ovarian cancer, accounting for approximately $90 \%$ of SOCs. ${ }^{3}$ No precancerous lesions were detected and were often found with extensive pelvic spread and high malignancy. ${ }^{4}$ LGOS is a type I ovarian cancer that accounts for approximately $10 \%$ of ovarian serous adenocarcinomas. ${ }^{3}$ These tumors grow slowly and are not sensitive to chemotherapy, but the clinical diagnosis is early, and the prognosis is satisfactory. ${ }^{5}$

During clinical diagnosis and treatment, the patients often asked gynecological oncologists: "If I have surgery and chemotherapy, how long will I live?" The AJCC staging system for ovarian cancer currently used internationally does not provide a good answer. The doctor has the ability to predict the probability of certain outcomes, which could modify the medical practice models and clinical decisions. Nomograms are statistics-based predictive tools that integrate pivotal predictive factors and are widely used to quantify risks and evaluate the prognosis of several cancer types. ${ }^{4,6,7}$ However, nomograms for patients with SOC have not yet been developed to the best of our knowledge. In this study, we aimed to construct nomograms using data extracted from the Surveillance, Epidemiology, and End Results (SEER) ${ }^{8}$ database to predict the prognosis of patients with SOC. Then, the model was verified externally to determine whether it provides accurate predictions of patient survival compared to the currently available staging systems.

\section{Materials and Methods \\ Data Sources and Extraction}

The training cohort of ovarian cancer patients was obtained from the SEER database, consisting of cancer patients' data from 18 regional registries, covering approximately $34.6 \%$ of the total United States population. ${ }^{9}$ The cases of ovarian cancer (International Classification of Diseases [ICD]-O-3, primary site, C56.9: ovary) diagnosed between 2010 and 2016 were selected. The ICD-O-3 morphology codes "8441-8442 and 8460-8463" were used to identify women with SOC. ${ }^{10}$ Relevant information was extracted using SEER*Stat software version 8.3.6.

A retrospective study was conducted for the external validation cohort of patients who underwent cytoreductive surgery for ovarian tumors between January 2009 and June 2015 at Guangxi Medical University Tumor Hospital and Guangxi Zhuang Autonomous Region
People's Hospital in Guangxi Province, China. All patients underwent satisfactory cytoreductive surgery with residual tumor diameter $<1 \mathrm{~cm}$ and paclitaxel combined with platinum chemotherapy.

\section{Inclusion and Exclusion Criteria}

The inclusion criteria for this study were as follows: no history of previous anticancer therapy, no history of other malignancies, satisfactory cytoreductive surgery, a pathological diagnosis of SOC (including LGOS and HGOS), the stage was not limited, at least three courses of paclitaxel combined with platinum-based intravenous chemotherapy were administered after surgery, and there was no age or race restriction. The exclusion criteria were as follows: no primary tumor, no histological confirmation, survival time $<1$ month, no surgery, no chemotherapy, and cases with incomplete information.

\section{Study Design and Ethics}

The final analysis consisted of 8201 individuals, of which the training cohort extracted from the SEER database $(n=6957)$ was used for model development, and the remaining validation samples were used for external validation of model ( $\mathrm{n}=1244)$. This study was approved by the ethics committee, and all patients signed the informed consent before surgery and chemotherapy.All procedures performed in studies involving human participants were in accordance with the ethical standards of the institutional and/or national research committee and with the 1964 Helsinki declaration and its later amendments or comparable ethical standards.

\section{Follow-Up}

The clinicopathological information and follow-up data were collected by four gynecologists with unified training. To ensure the accuracy of data entry, two specially trained gynecologists input the same medical records and established a database after checking the doubtful parameters. The SEER program included demographic data, stage of cancer at the time of diagnosis, and treatment information at the time of follow-up.

\section{Variates and Outcomes}

Variables were grouped to match with nomograms according to the clinical situation. The following factors were assessed at diagnosis: age $(<50,50-59,60-69,70-79$, and $\geq 80$ years), grade (LGOS or HGOS), and AJCC stage (I, II, III, or IV). OS was used as the primary endpoint and 
defined as the time from diagnosis to death or the last follow-up.

\section{Statistical Analysis}

We used standard model development and validation methods on training and external validation cohorts. ${ }^{11}$ The primary endpoint was OS. The categorical variables are shown as frequencies and proportions. The comparisons of clinicopathological characteristics between the training and validation cohorts were performed using the chi-squared test or Fisher's exact test. Continuous variables were compared using the $t$-test or Mann-Whitney $U$-test for variables with an abnormal distribution. Next, univariate and multivariate analysis was conducted via Cox proportional hazard regression models in the training cohort to analyze the different prognostic variables associated with OS and construct a nomogram. Those with a P-value $<0.05$ by univariate analysis were included in the multivariate analysis.

A nomogram was drawn using the RMS26 package in R version 3.6.2 (www.r-project.org; R Foundation, Vienna, Austria). The performance of the nomogram was validated internally and externally in the training and validation cohorts, respectively. The receiver operating characteristic (ROC) was used to evaluate the discriminative abilities of the nomograms. The larger the ROC, the more accurate the prognostic prediction. ${ }^{12}$ Calibration curves (1000 bootstrap resamples) were generated to test the consistency between the predicted and actual 3- and 5-year OS. Moreover, the whole cohort was regrouped into low- and high-risk groups, according to the median risk score predicted from the nomogram. Kaplan-Meier analysis and the Log rank test were used to explore the survival differences between the risk subgroups.

All statistical analyses were performed using SPSS version 25.0 (IBM Corp., Armonk, NY, USA) and $\mathrm{R}$ software version 3.6.2. P-values $<0.05$ indicated statistical significant.

\section{Results}

\section{Patient Characteristics}

The patient characteristics in the training $(n=6957)$ and validation $(n=1124)$ cohorts are listed in Table 1. A total of 8201 women with SOC were identified. As shown in Table 1 , the majority of the cases $(>85 \%)$ were diagnosed in women $\geq 50$-years-old. About $84.4 \%$ of patients had HGOS, of which $77.8 \%$ were at an advanced stage.
Table I Demographics and Clinicopathological Characteristics of Patients with SOC

\begin{tabular}{|c|c|c|c|c|c|c|}
\hline \multirow[t]{2}{*}{$\begin{array}{l}\text { Demographic or } \\
\text { Characteristic }\end{array}$} & \multicolumn{2}{|c|}{$\begin{array}{l}\text { All } \\
\text { Patients } \\
(n=820 I)\end{array}$} & \multicolumn{2}{|c|}{$\begin{array}{l}\text { Training } \\
\text { Cohort } \\
(n=6957)\end{array}$} & \multicolumn{2}{|c|}{$\begin{array}{l}\text { Validation } \\
\text { Cohort } \\
(n=\mid 244)\end{array}$} \\
\hline & $\mathbf{n}$ & $\%$ & $\mathbf{n}$ & $\%$ & $\mathbf{n}$ & $\%$ \\
\hline \multicolumn{7}{|l|}{ Age, years } \\
\hline$<50$ & 1079 & 13.2 & 1014 & 14.5 & 65 & 5.2 \\
\hline $50-59$ & 2163 & 26.3 & 1803 & 25.9 & 360 & 28.9 \\
\hline $60-69$ & 2813 & 34.3 & 2187 & 31.4 & 626 & 50.3 \\
\hline $70-79$ & 1576 & 19.2 & 1400 & 20.1 & 176 & 14.1 \\
\hline$\geq 80$ & 570 & 7.0 & 553 & 7.9 & 17 & 1.5 \\
\hline \multicolumn{7}{|l|}{ Grade } \\
\hline LGOS & 1276 & 15.6 & 728 & 10.5 & 548 & 44.1 \\
\hline HGOS & 6925 & 84.4 & 6229 & 89.5 & 696 & 55.9 \\
\hline \multicolumn{7}{|l|}{ AJCC stage } \\
\hline I & 960 & 11.7 & 684 & 9.8 & 276 & 22.2 \\
\hline II & 864 & 10.5 & 703 & 10.2 & 161 & 12.9 \\
\hline III & 4418 & 53.9 & 3730 & 53.6 & 688 & 55.3 \\
\hline IV & 1959 & 23.9 & 1840 & 26.4 & 119 & 9.6 \\
\hline
\end{tabular}

Abbreviations: LGOS, low-grade ovarian serous cancer; HGOS, high-grade ovarian serous cancer; AJCC, American Joint Committee on Cancer staging system (7th edition).

\section{Independent Prognostic Factors in the Training Cohort}

Univariate analysis of the training cohort showed that age, grade, and AJCC stage were significant risk factors for OS $(\mathrm{P}<0.05)$ (Table 2). The independent prognostic factors in the final model were identified by multivariate analysis, wherein the three factors, age, grade, and AJCC stage, were independent and significantly associated with OS (Table 2).

\section{Constructing Nomograms for OS}

Prognostic nomograms for predicting 3- and 5-year OS were constructed independently based on the prognostic variables from the training cohort. The complex Cox regression formulas were transformed into visual graphics. The line segment corresponding to each variable was marked with a scale that represented the value range of the variable, and the length reflected the contribution of the factor to the outcome. The nomograms demonstrated that the AJCC stage contributed to the OS of SOC patients. Nomograms are commonly used to estimate the prognosis in oncology. Each variable was assigned a score of $0-100$. To easily use the nomograms, one can draw a vertical line upwards from the specific points of each predictor to the "point" lines and then add up the corresponding points. ${ }^{13}$ Then, a straight line was 
Table 2 Univariate and Multivariate Cox Regression Analysis for OS in the Training Cohort

\begin{tabular}{|c|c|c|c|c|c|c|}
\hline \multirow{2}{*}{ Variables } & \multicolumn{3}{|c|}{ Univariate Analysis } & \multicolumn{3}{|c|}{ Multivariate Analysis } \\
\hline & HR & $95 \% \mathrm{Cl}$ & P-value & HR & $95 \% \mathrm{Cl}$ & P-value \\
\hline \multicolumn{7}{|l|}{ Age, years } \\
\hline$<50$ & I & & & I & & \\
\hline $50-59$ & 1.213 & $1.029-1.430$ & 0.021 & 1.142 & $0.969-1.347$ & 0.113 \\
\hline $60-69$ & 1.568 & $1.342-1.833$ & $<0.001$ & 1.437 & $1.230-1.680$ & $<0.001$ \\
\hline $70-79$ & 2.255 & $1.922-2.646$ & $<0.001$ & 1.959 & $1.668-2.300$ & $<0.001$ \\
\hline$\geq 80$ & 4.014 & $3.362-4.793$ & $<0.001$ & 3.934 & $3.292-4.702$ & $<0.001$ \\
\hline \multicolumn{7}{|l|}{ Grade } \\
\hline LGOS & I & & & I & & \\
\hline HGOS & 1.940 & $1.637-2.300$ & $<0.001$ & 1.393 & $1.173-1.653$ & $<0.001$ \\
\hline \multicolumn{7}{|c|}{ AJCC_stage } \\
\hline I & I & & & I & & \\
\hline II & 2.261 & $|.654-3.09|$ & $<0.001$ & 2.065 & $1.509-2.824$ & $<0.001$ \\
\hline III & 4.280 & $3.293-5.562$ & $<0.001$ & 4.124 & $3.169-5.367$ & $<0.001$ \\
\hline IV & 7.521 & $5.772-9.800$ & $<0.001$ & 6.909 & $5.293-9.018$ & $<0.001$ \\
\hline
\end{tabular}

Abbreviations: LGOS, low-grade ovarian serous cancer; HGOS, high-grade ovarian serous cancer; AJCC, American Joint Committee on Cancer staging system (7th edition); HR, hazard ratio.

drawn from the total points axis to the 3- and 5-year survival axis so that the surgeons could predict the probability of 3- and 5-year survival (Figure 1).

\section{Validation of the Nomograms}

The nomograms were externally validated. C-statistic, ROC curves, and calibration curves were used to validate the model. These indexes were used to evaluate the accuracy of prediction models.

\section{Values of the C-Statistic}

The C-statistic ranged from 0.5 (absence of discrimination) to 1.0 (perfect discrimination). Typically, if the C-statistic value was $>0.6$, the model had a good predictive value.

In the training cohort, the $\mathrm{C}$-statistic value for predicting OS was 0.689 (95\% confidence interval (CI): 0.677$0.701)$. In the validation cohort, the $\mathrm{C}$-statistic value for OS was 0.639 (95\% CI: 0.601-0.670). Thus, the C-statistic values of the training and validation sets were similar.

\section{ROC Curve Analyses}

The ROC curve for OS in the training and validation cohorts was 0.675 and 0.661 , respectively (Figure 2). The ROC curve values ranged from 0.5 (absence of discrimination) to 1.0 (perfect discrimination). Typically, if the ROC curve was $>0.6$, the model had a medium prediction value. The ROC curves of the training and validation sets were similar (Figure 2).

\section{Calibration Curves}

The calibration curves indicated an agreement between the nomogram predictions and the actual survival outcomes in the training and validation cohorts. The $\mathrm{x}$-axis represents the nomogram-predicted probability of each patient's 3- and 5-year OS rates, while the y-axis represents the actual 3and 5-year survival rates of each patient. If the blue line completely coincided with the black dashed line, the model was the most ideal. The agreement between the predicted vs observed probabilities was indicated by the calibration curves for 3- and 5-year OS (Figure 3).

\section{Risk Stratification of SOC Patients and Decision-Curve Analysis (DCA)}

A risk score of each variable was generated from the nomogram, and the total scores were calculated for all patients. The whole cohort was divided into low- and highrisk subgroups based on the median risk score. According to the survival curves shown in Figure 4A, significant differences were observed between the low- and highrisk groups for OS $(\mathrm{P}<0.001)$, which implied the risk stratification ability of the nomogram.

DCA indicated that the nomogram models make predictions and outperform the AJCC staging system (Figure 4B).

\section{Discussion}

In medical research, the tumor risk prediction models are used to predict the future incidence and prognosis of 


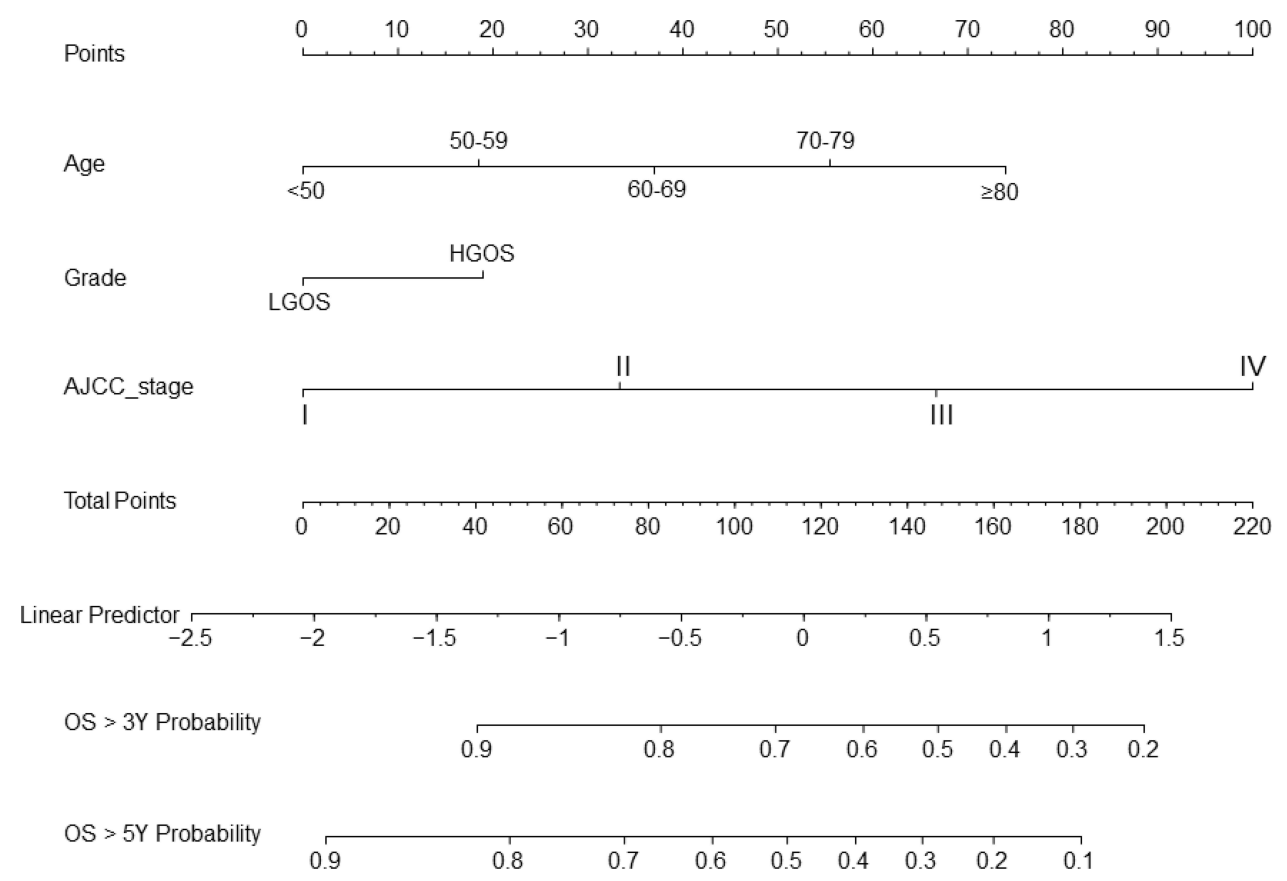

Figure I Nomograms for predicting the 3- and 5-year overall survival of patients with serous ovarian cancer in the training cohort.

Abbreviations: LGOS, low-grade ovarian serous cancer; HGOS, high-grade ovarian serous cancer; AJCC, American Joint Committee on Cancer staging system (7th edition); OS, Overall survival.

A

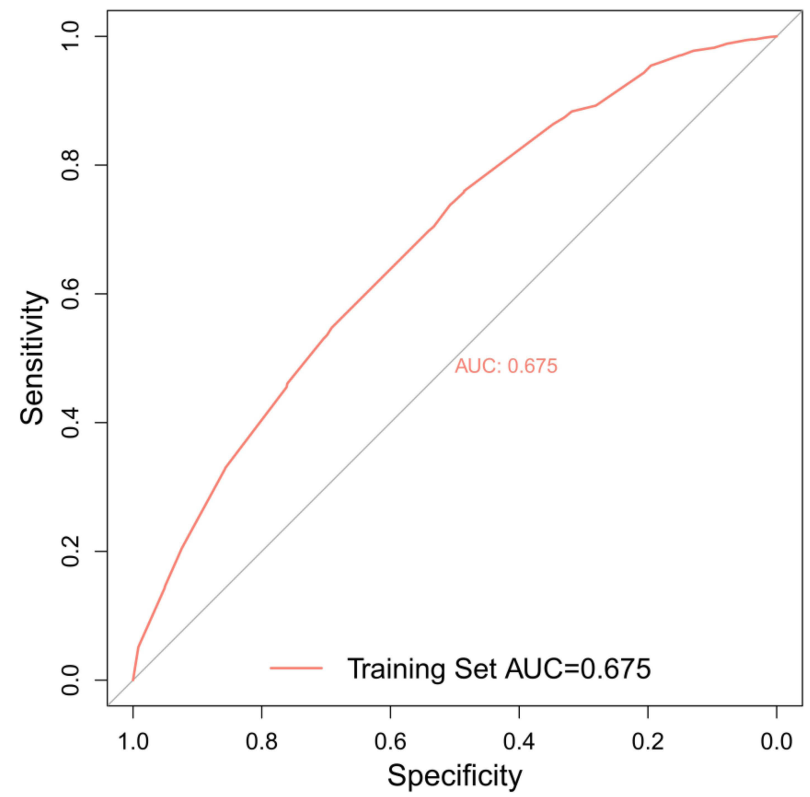

B

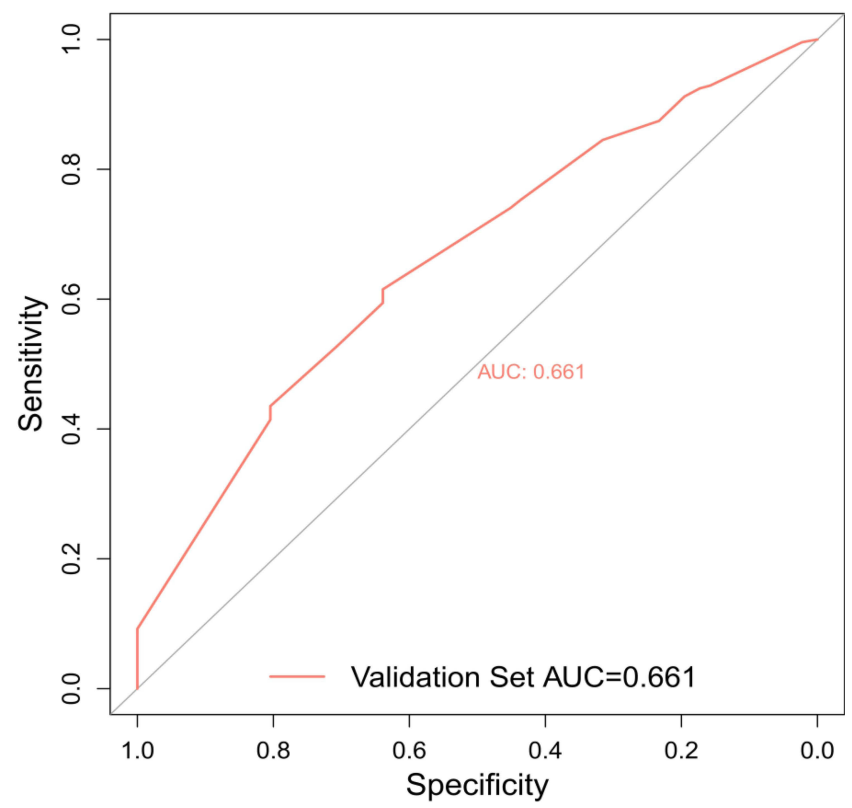

Figure 2 The ROC curve. The ability of the model to be measured by the AUC in the training cohort (A) and in the validation cohort (B).

certain tumors. Specifically, the nomograms represent a means to establish a statistical model of the quantitative correlation between multiple risk factors and tumor occurrence and/or prognosis. The purpose of such models was to inform the patients of the risk of onset or prognosis, screen high-risk groups, and help doctors make clinical decisions. In 2003, van Zee et $\mathrm{al}^{14}$ first proposed a nomogram model to predict the risk of non-sentinel lymph node metastasis in sentinel node-positive breast cancer. The advantage is that it provides individualized 
A

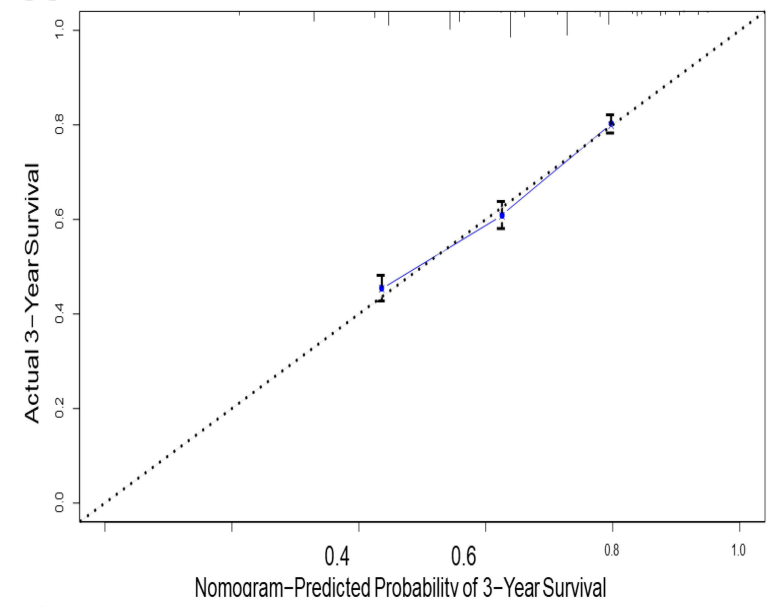

C

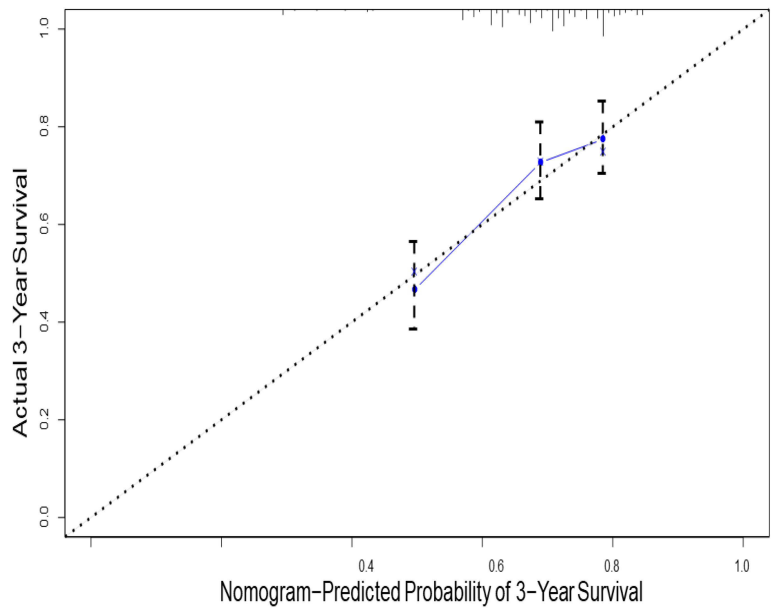

B

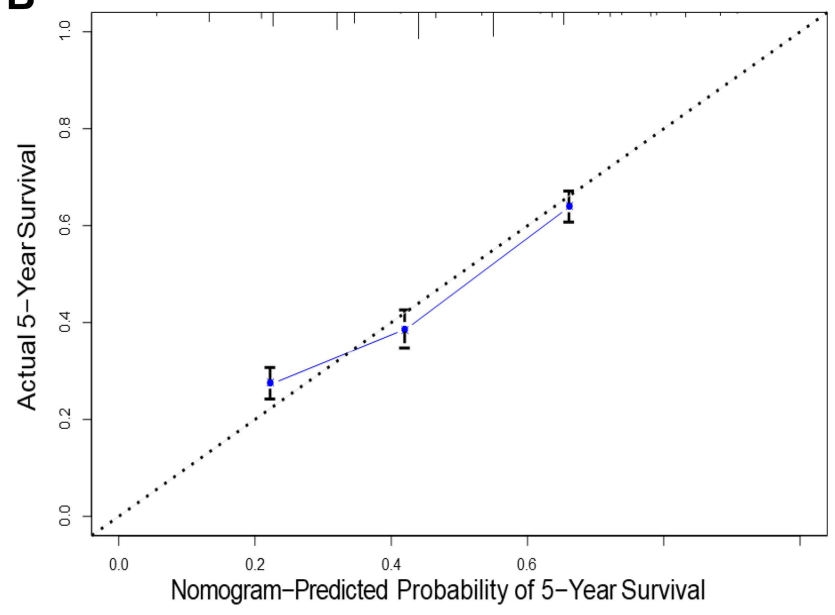

D

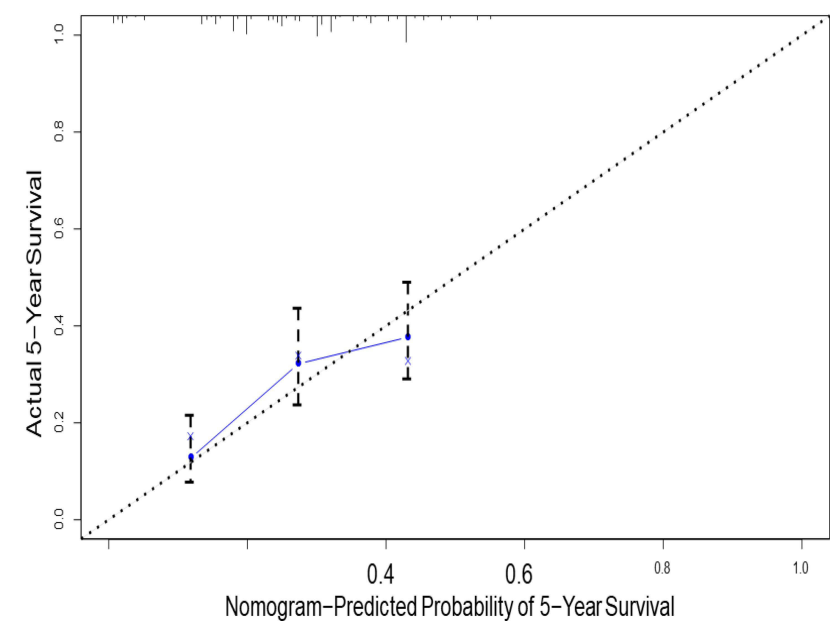

Figure 3 The calibration curves for predictions of 3-year (A) and 5-year OS (B) in the training cohort, and 3-year (C) and 5-year OS (D) in the validation cohort.

prognostic risk assessments in the form of intuitive graphics, which have a definite value in clinical practice and provide a reference for individualized clinical decisionmaking. Such predictive nomograms are widely used in clinical oncology. ${ }^{15-17}$ However, a lack of external verification was the common limitation of these studies. External validation of cohorts from other countries or prospective randomized clinical trials is essential to confirm a model's performance.

A subtype of EOC, SOC, accounts for approximately $85 \%$ of EOC diagnoses. Therefore, it is important to analyze the performance of nomograms independently with respect to the prognostic prediction of this EOC subtype. In this study, we used the external verification method and analyzed a training cohort of 6957 SOC patients from the SEER database and a validation cohort of 1244 SOC patients from two tertiary institutional hospitals to develop and validate the easy-to-use nomograms for predicting the 3- and 5-year OS. The current study identified the age, grade, and AJCC stage as independent predictors of OS. We also observed that the major predictors of improved OS were young age, early clinical stage, and well-differentiated grade, which is consistent with previous studies. ${ }^{18}$ In this study, the majority of cases $(>85 \%)$ were diagnosed in women aged >50-years-old; the older the patient, the worse the prognosis. Typically, older patients were likely to present poor survival outcomes due to low immune responses. ${ }^{19}$

Several scholars speculated that clinical staging is a critical factor that affects the prognosis of ovarian cancer. In the current study, $>70 \%$ of patients were diagnosed with advanced (III-IV) ovarian cancer. The later the clinical stage, the lower the 3- and 5-year OS rates. Patients with early Federation of Gynecology and Obstetrics (FIGO) staging could be more thoroughly removed after surgery, as the residual lesions were relatively small, 
A

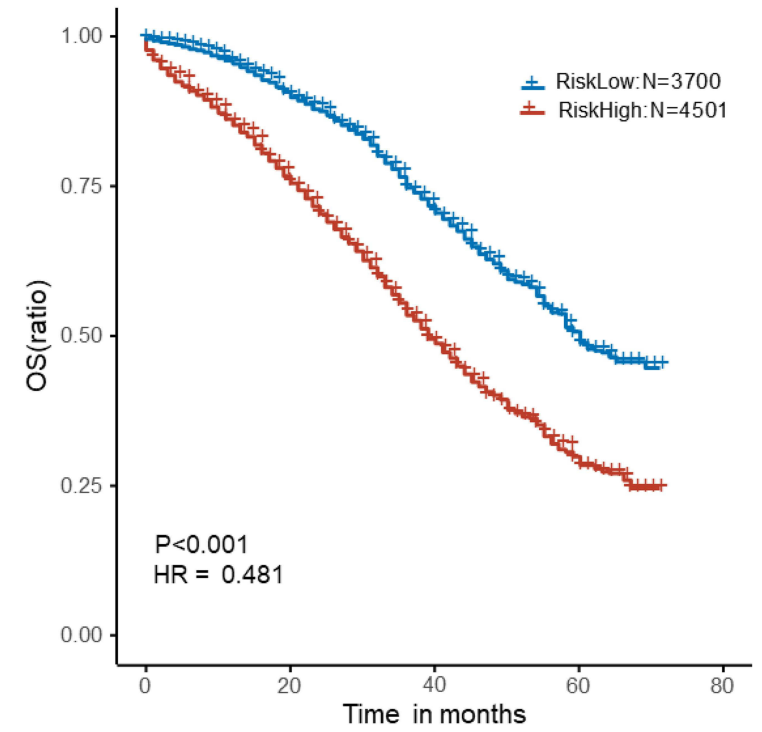

B

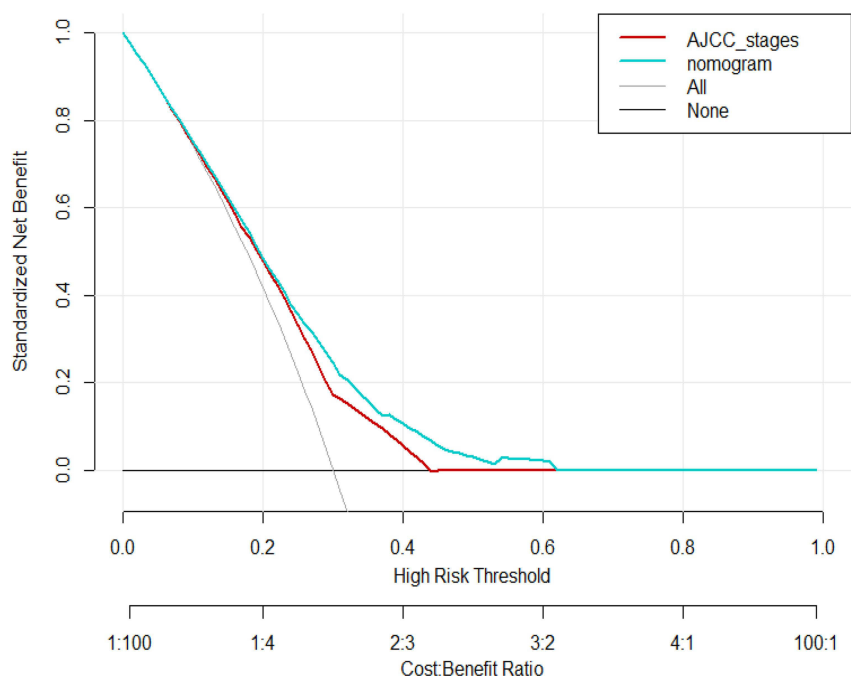

Figure 4 Kaplan-Meier cuves. OS for patients stratified by risk stratification system (A). DCA curve of the nomogram and AJCC stage for OS (B). Abbreviations: DCA, decision curve analysis; AJCC, American Joint Commission on Cancer; OS, overall survival.

chemotherapy-sensitive, and had a low risk of recurrence and metastasis, suggesting a good prognosis. Patients with late FIGO stage disease have tumor cells in the body that spread widely, making it difficult to implement complete surgical treatment. Patients with poor tolerance to chemotherapy have poor prognoses. Especially after stage IIIA, the later the stage, the higher the risk of death and the worse the prognosis. ${ }^{20}$

HGOS are the most common subtypes of EOC, and a majority of HGOS patients subsequently develop platinum resistance with relapse, indicating poor prognosis. $^{21}$ In this study, $84.4 \%$ of patients were HGOS. Based on the nomogram, the prognosis of these patients was worse than that of LGOS. Thus, it could be speculated that tumors with low histological grades have a high degree of malignancy, such as rapid disease progression and poor survival and prognosis due to adverse biological behavior (rapid cell proliferation, diffusion, and strong invasion). The higher the degree of tissue differentiation, the slower the proliferation of tumor cells in the body. The weak invasion of body tissues was associated with a low degree of malignancy, slow disease progression, sensitivity to chemotherapy, prolonged survival time, and improved prognosis.

Herein, we validated the accuracy of our nomograms using the $\mathrm{C}$-index, ROC analysis, and calibration curves in both the training and validation cohorts. The $\mathrm{C}$-index and ROC curves were $>0.6$ for OS in the external verification processes. The calibration curves also demonstrated the optimal performance of the nomograms. These findings indicated a discriminatory performance and calibration of our nomogram. Previous studies have used the SEER database to establish nomograms that could predict the prognosis of EOC. For example, Wang et al developed and internally validated the nomograms to predict OS and cancer-specific survival (CSS) of EOC patients, achieving a C-index of 0.733 for OS and 0.747 for $\mathrm{CSS}^{22}$ Chen et al constructed and validated the nomograms to predict OS and CSS in patients with ovarian clear cell carcinoma, achieving a C-index of 0.802 (95\% CI: 0.773-0.831) for OS and 0.802 (95\% CI: $0.769-0.835)$ for CSS. The nomograms were validated and found to be of good predictive value, which could aid in future clinical decisionmaking. $^{23}$

The traditional AJCC staging system does not accurately assess tumor prognosis because it only includes a limited subset of critical prognostic factors. Several studies have reported better performance of nomograms than conventional staging systems and have proposed such models as promising tools for evaluating prognosis. $\mathrm{Xu}$ et al developed and validated a nomogram based on log of odds between the number of positive lymph nodes and the number of negative lymph nodes to predict the OS and CSS for EOC patients, achieving a C-index of 0.757 (95\% CI: 0.746-0.768) for OS and 0.770 (95\% CI: 0.759-0.782) for CSS. Additionally, this model performed favorably 
compared to the currently used FIGO model with concordance indices of 0.699 (95\% CI: 0.688-0.710; $\mathrm{P}<0.05)$ and 0.719 (95\% CI: $0.709-0.730 ; \mathrm{P}<0.05$ ) for OS and CSS, respectively. This study suggested that the nomogram may be superior to the currently used FIGO staging system for predicting OS and CSS in postoperative EOC patients. ${ }^{24}$ The nomogram developed in the current study also showed better predictive accuracy for survival compared to the AJCC 7th staging system. It also enabled the risk stratification of patients, thus facilitating personalized treatment plans and follow-up schedules. For example, a patient $<50$-years-old with AJCC stage IV has poor prognosis based on the AJCC staging system. Therefore, the patient may give up treatment due to the economic burden. However, based on our nomogram, this patient might have a better prognosis with a 5 -year OS of $>50 \%$, which could promote the patient to cooperate with the doctor's treatment plan.

Our study has several advantages. First, we developed and validated nomograms using clinically important longterm oncological OS outcomes, which reduced the bias by scoring the model performance using high-quality data based on the large sample sizes from SEER. Second, although some studies have established nomograms to predict the prognosis of EOC, the nomogram model of these studies has only undergone internal validation. In contrast, the present study adopted the method of external verification using cohorts from other countries to confirm the performance. Third, compared to the AJCC-stages, DCA curves in this study showed that our nomograms provided excellent clinical utility. All variables included in the SOC nomogram were obtained easily and applied in clinical practice. Furthermore, we aimed to establish a prediction model for general SOC patients with common characteristics, such that the results were not affected by ethnic and regional differences. Although the SEER database contains information from the US population, our external validation population was from China. The C-statistic and AUC values of the training and validation sets were similar, demonstrating the discriminatory power of the nomogram. It also showed that this nomogram was applicable to regions other than the USA, facilitating clinical application.

Nevertheless, the present study has several limitations. First, the included variables were simple, and detailed data, such as a family history of ovarian cancer, primary tumor diameter, positive lymph nodes, ascites cytological results, location of metastasis, chemotherapy regimens and cycles, sensitivity to chemotherapy, and genetic results, could have been included. Second, this study was based on retrospective data; hence, bias may have been introduced due to some undetected potential factors.

\section{Conclusion}

We used the SEER database to construct a nomogram of SOC prognosis by integrating various prognostic factors into a simple and intuitive tool and externally verifying the model in an Asian population to obtain universally applicable unbiased estimates. In the future study, we will include additional variables while constructing accurate predictive models of SOC prognoses.

\section{Data Sharing Statement}

- The datasets generated and/or analysed during the current study are available in the SEER database repository.

- The external validation datasets used and analysed during the current study are available from the corresponding author on reasonable request.

\section{Ethics Approval and Consent to Participate}

All procedures performed in studies involving human participants were in accordance with the ethical standards of the institutional and/or national research committee and with the 1964 Helsinki declaration and its later amendments or comparable ethical standards. This article does not contain any studies with animals performed by any of the authors. The present study was approved by the Ethics Committee of Guangxi Medical University Cancer Hospital and Ethics Committee of The People's Hospital of Guangxi Zhuang Autonomous Region. The information was accessed from the online database. Informed consent was obtained from all individual participants included in the study.

\section{Informed Consent}

Written informed consent was obtained from the patients.

\section{Author Contributions}

Y. L.: protocol/project development, data management and manuscript writing; R. Z.: data collection; L. C.: data collection and data analysis; J. Z.: data collection and data analysis; L. Y.: data management; L. L.: protocol development, manuscript writing and editing. All authors made substantial contributions to conception and design, acquisition of data, or analysis and interpretation of data; took part in drafting the article or revising it critically for important intellectual 
content; agreed to submit to the current journal; gave final approval of the version to be published; and agree to be accountable for all aspects of the work.

\section{Funding}

This research did not receive any specific grant from funding agencies in the public, commercial, or not-forprofit sectors.

\section{Disclosure}

The authors report no conflicts of interest in this work.

\section{References}

1. Singer G, Kurman RJ, Chang HW, et al. Diverse tumorigenic pathways in ovarian serous carcinoma. Am J Pathol. 2002;160:1223-1228. doi:10.1016/S0002-9440(10)62549-7

2. Shih IM, Kurman RJ. Ovarian tumorigenesis: a proposed model based on morphological and molecular genetic analysis. Am J Pathol. 2004;164:1511-1518. doi:10.1016/S0002-9440(10)63708-X

3. Plaxe SC. Epidemiology of low-grade serous ovarian cancer. $\mathrm{Am}$ J Obstet Gynecol. 2008;198:459.e451-458; discussion 459.e458-459. doi:10.1016/j.ajog.2008.01.035

4. Kurman RJ, ShihIe IM. Pathogenesis of ovarian cancer: lessons from morphology and molecular biology and their clinical implications. Int $J$ Gynecol Pathol. 2008;27:151-160. doi:10.1097/ PGP.0b013e318161e4f5

5. Schmeler KM, Gershenson DM. Low-grade serous ovarian cancer: a unique disease. Curr Oncol Rep. 2008;10:519-523. doi:10.1007/ s11912-008-0078-8

6. Pan X, Yang W, Chen Y, et al. Nomogram for predicting the overall survival of patients with inflammatory breast cancer: a SEER-based study. Breast. 2019;47:56-61. doi:10.1016/j.breast.2019.05.015

7. Hu JQ, Yu PC, Shi X, et al. Prognostic nomograms for predicting overall survival and cancer-specific survival of patients with major salivary gland mucoepidermoid carcinoma. $J$ Cancer. 2019;10:4380-4388. doi:10.7150/jca.27992

8. Surveillance, Epidemiology, and End Results (SEER). Program: incidence-SEER 18 regs custom data (with additional treatment fields), Nov 2018 Sub (1975-2016 varying) National Cancer Institute, DCCPS, Surveillance Research Program, released April 2019, based on the November 2018 submission. Available from: https://seer.cancer. gov/. Accessed December 30, 2021.

9. Peres LC, Cushing-Haugen KL, Köbel M, et al. Invasive epithelial ovarian cancer survival by histotype and disease stage. $J$ Natl Cancer Inst. 2019;111:60-68. doi:10.1093/jnci/djy071
10. Royston P, Moons KG, Altman DG, et al. Prognosis and prognostic research: developing a prognostic model. BMJ. 2009;338:b604. doi:10.1136/bmj.b604

11. Huitzil-Melendez FD, Capanu M, O'Reilly EM, et al. Advanced hepatocellular carcinoma: which staging systems best predict prognosis? J Clin Oncol. 2010;28:2889-2895. doi:10.1200/ JCO.2009.25.9895

12. Wang Y, Li J, Xia Y, et al. Prognostic nomogram for intrahepatic cholangiocarcinoma after partial hepatectomy. $J$ Clin Oncol. 2013;31:1188-1195. doi:10.1200/JCO.2012.41.5984

13. Balachandran VP, Gonen M, Smith JJ, et al. Nomograms in oncology: more than meets the eye. Lancet Oncol. 2015;16:e173-180. doi:10.1016/S1470-2045(14)71116-7

14. Van Zee KJ, Manasseh DM, Bevilacqua JL, et al. A nomogram for predicting the likelihood of additional nodal metastases in breast cancer patients with a positive sentinel node biopsy. Ann Surg Oncol. 2003;10:1140-1151. doi:10.1245/ASO.2003.03.015

15. Moreau JT, Hankinson TC, Baillet S, et al. Individual-patient prediction of meningioma malignancy and survival using the surveillance, epidemiology, and end results database. NPJ Digit Med. 2020;3:12. doi:10.1038/s41746-020-0219-5

16. Yan $\mathrm{H}$, Wei $\mathrm{X}, \mathrm{Wu} \mathrm{A}$, et al. Nomograms for predicting overall and cancer-specific survival in patients with papillary renal cell carcinoma: a population-based study using SEER database. Transl Androl Urol. 2020;9:1146-1158. doi:10.21037/tau-19-807

17. Zi H, Gao L, Yu Z, et al. Nomograms for predicting long-term overall survival and cancer-specific survival in patients with primary urethral carcinoma: a population-based study. Int Urol Nephrol. 2020;52:287-300. doi:10.1007/s11255-019-02314-y

18. Zhang W, Zhang W, Lin Z, et al. Survival outcomes of patients with primary breast cancer following primary ovarian cancer. Med Sci Monit. 2019;25:3869-3879. doi:10.12659/MSM.914163

19. Pawelec G. Does patient age influence anticancer immunity? Semin Immunopathol. 2019;41:125-131. doi:10.1007/s00281-018-0697-6

20. Ge L, Li N, Yuan GW, et al. Nedaplatin and paclitaxel compared with carboplatin and paclitaxel for patients with platinum-sensitive recurrent ovarian cancer. Am J Cancer Res. 2018;8:1074-1082.

21. van Zyl B, Tang D, Bowden NA. Biomarkers of platinum resistance in ovarian cancer: what can we use to improve treatment. Endocr Relat Cancer. 2018;25:R303-r318. doi:10.1530/ERC-17-0336

22. Wang R, Xie G, Shang L, et al. Development and validation of nomograms for epithelial ovarian cancer: a SEER population-based, real-world study. Future Oncol. 2021;17:893-906. doi:10.2217/fon2020-0531

23. Chen Q, Wang S, Lang JH. Development and validation of nomograms for predicting overall survival and cancer-specific survival in patients with ovarian clear cell carcinoma. $J$ Ovarian Res. 2020;13:123. doi:10.1186/s13048-020-00727-3

24. Xu XL, Cheng H, Tang MS, et al. A novel nomogram based on LODDS to predict the prognosis of epithelial ovarian cancer. Oncotarget. 2017;8:8120-8130. doi:10.18632/oncotarget.14100
International Journal of General Medicine

\section{Publish your work in this journal}

The International Journal of General Medicine is an international, peer-reviewed open-access journal that focuses on general and internal medicine, pathogenesis, epidemiology, diagnosis, monitoring and treatment protocols. The journal is characterized by the rapid reporting of reviews, original research and clinical studies across all disease areas. The manuscript management system is completely online and includes a very quick and fair peer-review system, which is all easy to use. Visit http://www.dovepress.com/ testimonials.php to read real quotes from published authors. 